\title{
Fractura triplanar de tibia proximal
}

\section{Triplanar Fracture of Proximal Tibia}

\author{
Antonio Gámez-Martínez ${ }^{1}$ Jade García-Espinosa ${ }^{1} \quad$ Alberto Martínez-Martínez ${ }^{1}$ *
}

${ }^{1}$ Unidad de Gestión clínica de Diagnóstico por Imagen, Complejo Hospitalario Universitario de Granada, Granada, España

Rev Argent Radiol 2018;82:137-138.

Estimados Editores,

Las fracturas triplanares de la tibia distal son una entidad bien conocida, sin embargo, son excepcionales en otras localizaciones. ${ }^{1-4}$

Existen muy pocos casos publicados en la literatura de ese tipo de fractura que se sitúen en la tibia proximal. Aunque sea una patología excepcional, debe tenerse en cuenta por la alta posibilidad de presentar complicaciones y secuelas, dada su proximidad con la articulación de la rodilla y con estructuras vasculares y nerviosas adyacentes. ${ }^{1}$

Nuestro objetivo es realizar una breve revisión de las características de las fracturas triplanares, en este caso, situadas en una localización excepcional como lo es la tibia proximal.

Remitimos el caso de un paciente varón de 17 años de edad, sin antecedentes de interés, que tras una caída
Address for correspondence Alberto Martínez-Martínez, MD, Unidad de Gestión clínica de Diagnóstico por Imagen, Complejo Hospitalario Universitario de Granada, C/ Puerta Nueva 39, Hornos, 23292, Jaén, Granada, España (e-mail: rralbert.martinez@gmail.com).

circulando en bicicleta sufrió una contusión directa en ambas rodillas. En la exploración clínica, se objetivó una limitación para la flexo-extensión completa y choque rotuliano bilateral. La radiografía simple mostró múltiples trazos de fractura en tibia proximal derecha (-Fig. 1), por lo que se solicitó una tomografía computarizada (TC) para una mejor caracterización de los hallazgos y planificación quirúrgica.

En la TC se pudo apreciar una fractura en los tres planos del espacio con afectación epifisaria anterior y medial, trazo fisario horizontal y un trazo oblicuo metafisario medial posterior, todo ello compatible con las características de una fractura triplanar (-Fig. 2). Se intervino quirúrgicamente al paciente, realizándose una reducción abierta y osteosíntesis con placa en la parte posterior de la tibia, así como una osteosíntesis percutánea del fragmento
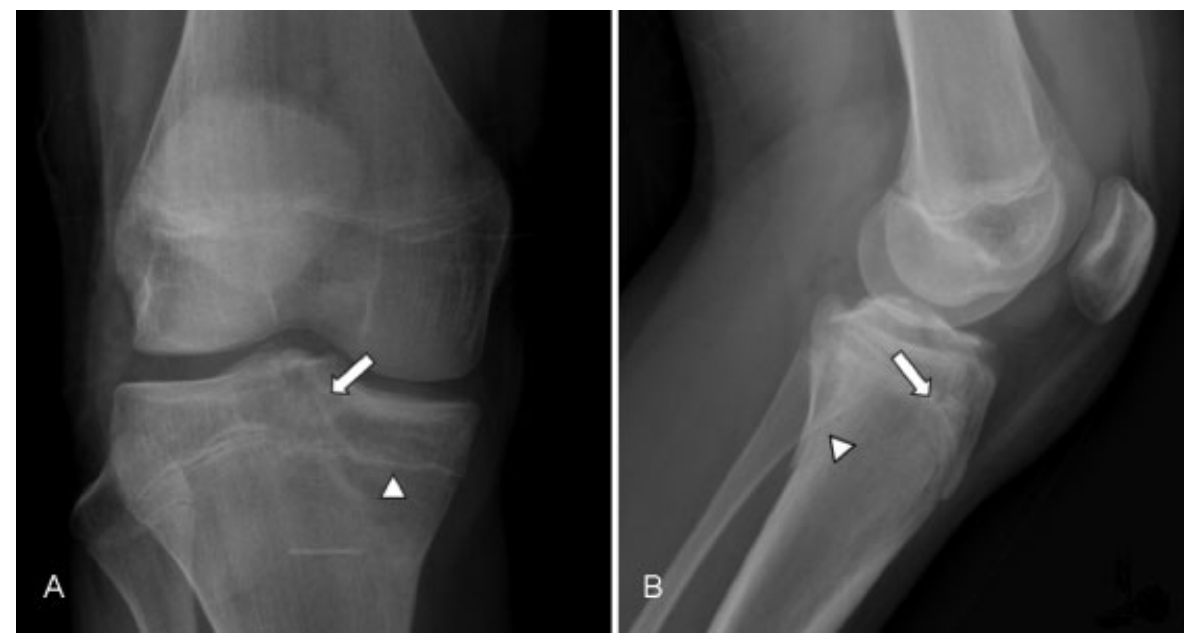

Fig. 1 Radiografía simple (Rx) de frente (A) de rodilla derecha con trazo de fractura vertical epifisario (flecha) y trazo horizontal fisario (punta de flecha). (B) Rx en proyección lateral donde se identifican trazos de fractura que afectan a la meseta tibial (flecha) y metáfisis de tibia proximal (punta de flecha).

received

July 2, 2017

accepted

September 20, 2017

published online

May 17, 2018
DOI https://doi.org/

10.1055/s-0038-1639572.

ISSN 1852-9992.
Copyright $\odot$ 2019, Sociedad Argentina de Radiología. Publicado por Thieme Revinter Publicações Ltda., Rio de Janeiro, Brazil. Todos los derechos reservados.
License terms

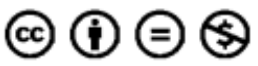



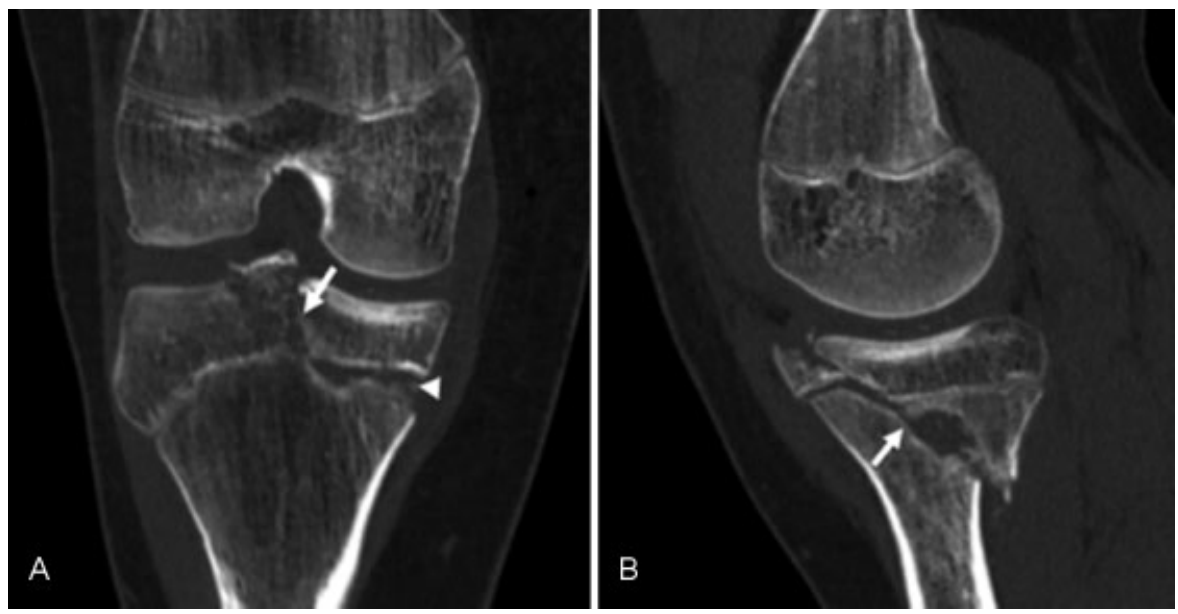

Fig. 2 Tomografía Computada (TC) de rodilla, plano coronal (A) donde se observa un trazo de fractura vertical epifisario medial anterior (flecha) y un trazo horizontal fisario (punta de flecha). TC de rodilla plano sagital (B) con trazo oblicuo metafisario medial posterior (flecha).

epifisario con dos tornillos. A los 3 meses de evolución y tras tratamiento rehabilitador, la evolución clínica y radiológica fue favorable, con inicio de marcha y carga.

Las fracturas triplanares fueron descritas por primera vez por Bartl ${ }^{5}$ en 1957, refiriéndose a un tipo de fracturas con componentes epifisario y metafisario. El término "triplano" hace referencia a la orientación de los trazos de fractura implicados, disponiéndose en los tres planos del espacio transversal, sagital y coronal; normalmente se producen en traumatismos de alta energía. El mecanismo de producción en la tibia proximal es poco conocido, siendo en la tibia distal secundarias a fuerzas de rotación externa sobre un pie en flexión plantar. ${ }^{2}$ Son más frecuentes en pacientes adolescentes y suelen afectar al cartílago de crecimiento, por lo que resulta necesario un seguimiento posterior para evitar deformaciones futuras. ${ }^{2-4}$

Pueden asociarse a lesión de la arteria poplítea y nervio peroneo debido a la cercanía de esas estructuras, así como al síndrome compartimental, siendo necesario un diagnóstico adecuado y un seguimiento estrecho. ${ }^{1}$ La fractura triplanar de la tibia proximal es una lesión excepcional y grave que conlleva una alta morbilidad. Es necesario un diagnóstico preciso de la misma y reconocerla como una entidad propia para establecer un adecuado manejo terapéutico.
Confidencialidad de los datos

Los autores declaran que han seguido los protocolos de su centro de trabajo sobre la publicación de datos de pacientes y que todos los pacientes incluidos en el estudio han recibido información suficiente y han dado su consentimiento informado por escrito para participar en dicho estudio.

Conflicto de intereses

Los autores del trabajo declaran no tener ningún conflicto de intereses.

\section{Bibliografia}

1 Nowicki PD, Ebraheim NA, Gomez CE, Rabenold J. Proximal tibia triplane fracture: A serious presentation of a serious injury. Inj Extra 2010;41:31-34

2 El-Karef E, Sadek HI, Nairn DS, Aldam CH, Allen PW. Triplane fracture of the distal tibia. Injury 2000;31(09):729-736

3 Kanellopoulos AD, Yiannakopoulos CK, Badras LS. Triplane fracture of the proximal tibia. Am J Orthop 2003;32(09):452-454

4 Rico-Peceroa J, Dwyera A. Fractura triplana de tobillo relacionada con fractura tibial homolateral en un adolescente. Caso clínico. Rev Esp Cir Ortop Traumatol 2009;53:254-256

5 Bartl R. Die traumatische Epiphysenlosung am distalen ende des Scheinbein esund des Wadenbeines. Hefte Unfallheilkd 1957; 54:228-257 